\title{
Synthesis and reactions of benzotriazolyl epoxides
}

\author{
Alan R. Katritzky, ${ }^{*}$ Rexiat Maimait, Anna Denisenko, and Sergey N. Denisenko \\ Center for Heterocyclic Compounds, Department of Chemistry, University of Florida, \\ Gainesville, Florida 32611-7200 \\ E-mail:katritzky@chem.ufl.edu
}

Dedicated to Professor Miha Tišler on the Occasion of his $75^{\text {th }}$ birthday

(received 10 Apr 01; accepted 03 Dec 01; published on the web 11 Dec 01)

\begin{abstract}
Substituted vinylbenzotriazoles were efficiently converted to benzotriazolyl epoxides by dimethyldioxirane. The behavior of these epoxides toward nucleophiles and strong bases was investigated.
\end{abstract}

Keywords: Benzotriazole, epoxides, dimethyldioxirane, lithiation

\section{Introduction}

A recent study demonstrated the dimethyldioxirane (DMD) conversion of 1alkylbenzotriazoles into 3-alkylbenzotriazole 1-oxides. ${ }^{1}$ We now report the efficient and almost quantitative low temperature epoxidation of vinylbenzotriazoles by dimethyldioxirane together with some reactions of the epoxides formed. 
Epoxides are important in organic synthesis due to their high reactivity. ${ }^{2,3}$ A series of $\alpha$ benzotriazolyl epoxides was previously synthesized in yields of 43-80\% from 1substituted 1(benzotriazolyl)alkenes by oxidation with $m$-CPBA and subsequently converted into the corresponding $\alpha$-hydroxy ketones. ${ }^{4}$ Benzotriazole groups are synthetically useful ${ }^{5}$ as: (i) activators for proton loss, ${ }^{6}$ (ii) cation stabilizers, (iii) good leaving groups and (iv) radical precursors. $^{7}$ Such properties of the benzotriazolyl group have not been studied extensively in oxiranes. We therefore examined possible reactions with nucleophiles, and the deprotonation of $\alpha$-benzotriazole substituted epoxides.

\section{Results and Discussion}

\section{Synthesis of $\alpha$-benzotriazolyl epoxides.}

A solution of DMD in $\mathrm{CH}_{2} \mathrm{Cl}_{2}(0.2-0.4 \mathrm{M})^{8,9}$ converted vinylbenzotriazoles 1a-d into the corresponding epoxides 2a-d; simple removal of the solvent gave the products in $96-99 \%$ yields (Scheme 1). Details for the syntheses of epoxides 2a-d are given in Table 1. The reactions of non-functionalized alkenes or those bearing electron donor groups were complete in less than an hour at $-20{ }^{\circ} \mathrm{C}$; but when $\mathrm{R}^{2}$ was a phenyl group, the reaction took $8-10 \mathrm{~h}$ at $0{ }^{\circ} \mathrm{C}$. Compound 1e, where both $\mathrm{R}^{1}$ and $\mathrm{R}^{2}$ were phenyl groups, was consumed only after $30 \mathrm{~h}$ at $0{ }^{\circ} \mathrm{C}$; $1 \mathrm{e}$ was the only vinylbenzotriazole to undergo non-stereoselective oxidation to give the corresponding epoxide 2e (52\%) along with the 1-benzotriazolyl 3-N-oxide substituted epoxide 3 (34\%) (Scheme 1).

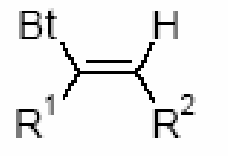

1a-e
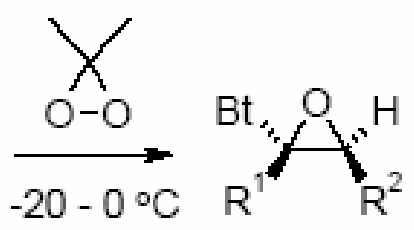

2a-e

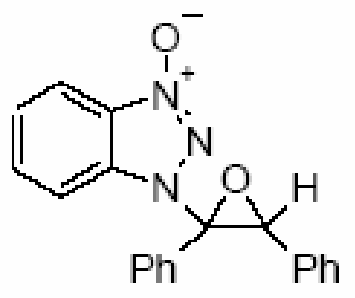

3

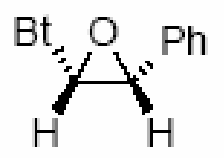

4

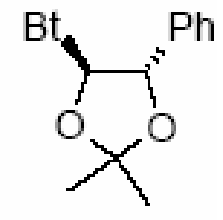

5

a: $R^{1}=R^{2}=H$

b: $\mathrm{R}^{1}=\mathrm{CH}_{3}, \mathrm{R}^{2}=\mathrm{H}$

c: $\mathrm{R}^{1}=\mathrm{H}, \mathrm{R}^{2}=\mathrm{CH}_{3}$

d: $R^{1}=H, R^{2}=P h$

e: $R^{1}=R^{2}=P h$

\section{Scheme 1}<smiles></smiles> 
Table 1. Synthesis of $\alpha$-benzotriazolyl epoxides 2

\begin{tabular}{cccccc}
\hline Epoxides & $\mathrm{R}_{1}$ & $\mathrm{R}_{2}$ & Time $(\mathrm{h})$ & $\mathrm{Temp}\left({ }^{\circ} \mathrm{C}\right)$ & Yield (\%) \\
\hline 2a & $\mathrm{H}$ & $\mathrm{H}$ & 0.5 & -20 & 99 \\
2b & $\mathrm{CH}_{3}$ & $\mathrm{H}$ & 0.1 & -20 & 97 \\
2c & $\mathrm{H}$ & $\mathrm{CH}_{3}$ & 0.1 & -20 & 99 \\
2d & $\mathrm{H}$ & $\mathrm{Ph}$ & 10 & 0 & 99 \\
2e & $\mathrm{Ph}$ & $\mathrm{Ph}$ & 30 & 0 & 52 \\
\hline
\end{tabular}

Compounds 1a-d reacted with $\mathrm{DMD}$ exclusively at the $\mathrm{C}=\mathrm{C}$ bond to give epoxides, but not at the N-3 atom of the benzotriazole ring. This can be explained by the relative energies of the two MO's localized mainly on the $\mathrm{C}=\mathrm{C}$ bond and on the N-3 atom. According to a study of the electronic structure of 1-vinyl- $1 H$-benzotriazoles, ${ }^{10}$ the energy of the $\mathrm{MO}$ of the $\mathrm{C}=\mathrm{C}$ bond is higher than that of those localized on $\mathrm{N}-3$. The order of these two MOs here is reversed compared to that of 1-allylbenzotriazole, which is oxidized to the $3-\mathrm{N}$-oxides. ${ }^{1}$

The stereochemistry of the olefins was preserved during oxidations below $0{ }^{\circ} \mathrm{C}$ : trans-olefins 1c and 1d gave trans-epoxides 2c and 2d, respectively. The trans-stereochemistry of the epoxides $2 \mathbf{c}$ and $2 \mathbf{d}$ was deduced from the vicinal coupling constant (1.5 Hz or less) that is in agreement with the literature data for $J$ values $(0-1.7 \mathrm{~Hz})$ for 1-aryl-2-phenyl epoxides. ${ }^{11}$ In the ${ }^{1} \mathrm{H}$ NMR spectrum of $\mathbf{2 c}$, the $\alpha-\mathrm{H}$ (with respect to $\mathrm{Bt}$ ) appeared as a doublet at $\delta 5.44$ with a coupling constant of $1.2 \mathrm{~Hz}$, while the $\beta-\mathrm{H}$ appeared as a doublet of quartets at $\delta 4.24(J=1.2$ and $5.4 \mathrm{~Hz}$ ), and the methyl group appeared as a doublet at $\delta 1.60(\mathrm{~J}=5.4 \mathrm{~Hz})$. In the ${ }^{1} \mathrm{H}$ NMR spectrum of 2d, two epoxide protons were observed at $\delta 5.11$ and $5.69 \mathrm{ppm}$ as doublets with a coupling constant of $1.5 \mathrm{~Hz}$.

A mixture of the cis (40\%) and trans (60\%) isomers of $\mathbf{1 d}$ reacted with 1.5 equiv of DMD to give three isolated products: the trans-epoxide $2 \mathbf{d}(52 \%)$, the cis-epoxide $4(28 \%)$ and the 1,3dioxolane 5 (1.4\%) (Scheme 1). The cis-stereochemistry of 4 was confirmed by the ${ }^{1} \mathrm{H}$ NMR spectrum, in which two doublets of integral intensity of $1 \mathrm{H}$ were observed at $\delta 5.82(\mathrm{~J}=2.6 \mathrm{~Hz})$ and $4.58(J=2.6 \mathrm{~Hz})$. In the ${ }^{1} \mathrm{H}$ NMR spectrum of 5 , two methyl groups appeared as singlets at $\delta$ 1.83 and 1.88; the dioxolane protons appeared as doublets at $\delta 6.40$ and 6.61 with a coupling constant of $6.1 \mathrm{~Hz}$. The trans-stereochemistry of 5 was assigned by comparing the ${ }^{1} \mathrm{H}$ NMR spectrum to literature data. ${ }^{12}$ The coupling constant is slightly smaller than that of a similar type of dioxolane $(J=8.5 \mathrm{~Hz})^{12}$ due to the presence of the electron-withdrawing benzotriazole group. 


\section{Deprotonation of $\alpha$-benzotriazolyl epoxides and subsequent reactions with electrophiles.}

Since Eisch and Galle $^{13}$ reported the deprotonation of $\alpha$-heterosubstituted epoxides, oxiranyllithium compounds have become important synthetic intermediates.Phenylsulphonyl ${ }^{14}$ and benzothiazolyl ${ }^{15}$ groups were used to stabilize the oxiranyl anion successfully in the functionalization of epoxides. Here we report the generation of oxiranyllithium species stabilized by a benzotriazole group and their reactions.

Treatment of the simplest benzotriazolyl epoxide 2a with LDA at $-78{ }^{\circ} \mathrm{C}$, followed by the addition of $\mathrm{MeI}$ failed to give any coupling product. When $\mathrm{Me}_{3} \mathrm{SiCl}$ was used at $-116{ }^{\circ} \mathrm{C}$, the anion generated in situ from 2a by LDA, was captured to give the expected product $\mathbf{6}$ in $30 \%$ isolated yield. This proved the formation of an oxiranyllithium species that is stabilized by benzotriazole. Treatment of epoxide 2d with fresh LDA at $-116{ }^{\circ} \mathrm{C}$, followed by the immediate addition of electrophile $\mathrm{PhCH}_{2} \mathrm{Br}$, afforded epoxide 8 in $51 \%$ yield along with the rearranged product 11 (Scheme 2). The generation of oxiranyllithium $\mathbf{7}$ from $\mathbf{2 d}$ by LDA is very fast at $116{ }^{\circ} \mathrm{C}$, which is stable at and below this temperature for about 5 to 20 minutes. As the temperature goes above $-110^{\circ} \mathrm{C}$, the anion starts to undergo rearrangement to give compound 11, which is the major product when the LDA is not freshly prepared. The rearrangement product 11 was the only product, when $2 \mathbf{d}$ was treated with LDA at $-116{ }^{\circ} \mathrm{C}$, and allowed to warm up to $20^{\circ} \mathrm{C}$. The oxiranyllithium 7 reacts with a strong electrophile $p$-toluoyl chloride to give coupling product 9 in $56 \%$ yield; it also reacts with a hindered ketone, benzophenone, to give oxiranyl alcohol $\mathbf{1 0}$ in 52\% yield, as shown in Table 2. 


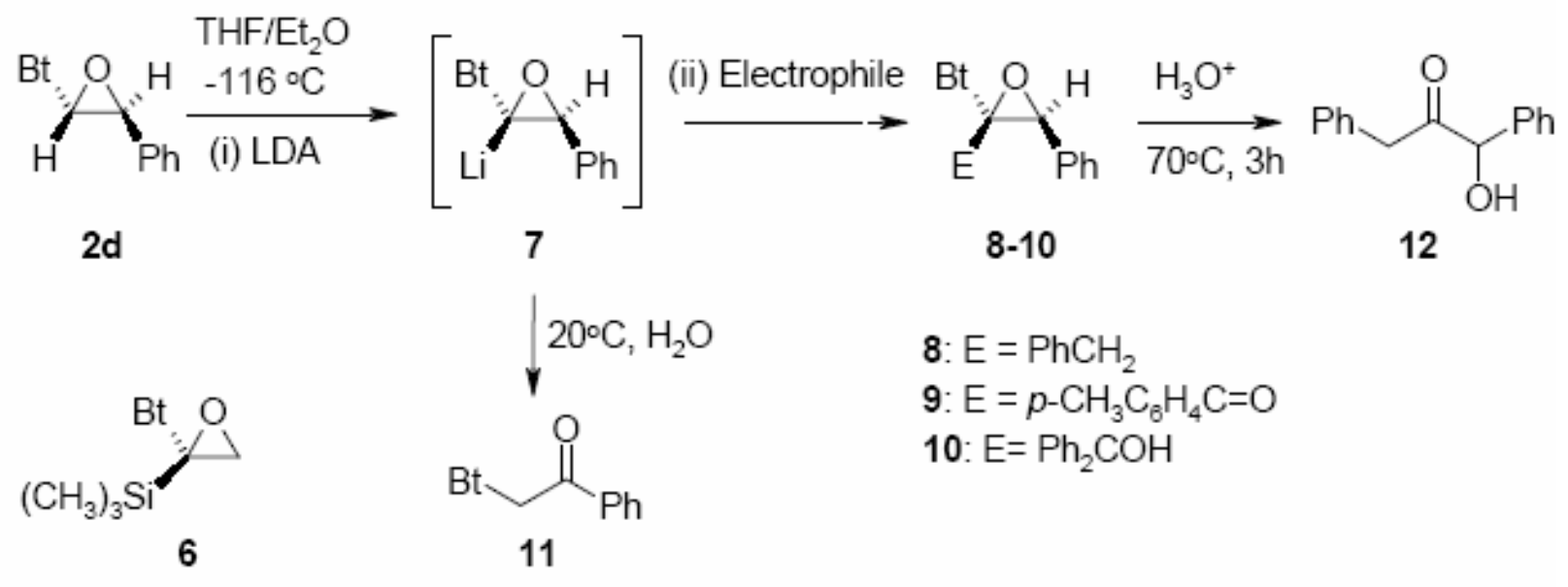

Scheme 2

Table 2. The coupling reactions of oxiranyllithium 7 from $\mathbf{2 d}$ with electrophiles

\begin{tabular}{cccc}
\hline Product & Electrophile & $\mathrm{E}$ & Yield (\%) \\
\hline $\mathbf{8}$ & $\mathrm{PhCH}_{2} \mathrm{Br}$ & $\mathrm{PhCH}_{2}$ & 51 \\
$\mathbf{9}$ & $p-\mathrm{CH}_{3} \mathrm{C}_{6} \mathrm{H}_{4} \mathrm{COCl}$ & $p-\mathrm{CH}_{3} \mathrm{C}_{6} \mathrm{H}_{4} \mathrm{C}=\mathrm{O}$ & 56 \\
$\mathbf{1 0}$ & $\mathrm{Ph}_{2} \mathrm{C}=\mathrm{O}$ & $\mathrm{Ph}_{2} \mathrm{COH}$ & 52 \\
\hline
\end{tabular}

Reactions with nucleophiles. We next examined the possible replacement of the benzotriazole group in epoxides 2 by nucleophiles, which could be activated by an $\alpha$-oxygen atom. The reaction of Grignard reagent $\mathrm{PhCH}_{2} \mathrm{MgCl}$ with 2a or $2 \mathbf{d}$ at -30 to $-18{ }^{\circ} \mathrm{C}$ gave complex mixtures. When trisubstituted epoxide 8 was treated with $\mathrm{PhCH}_{2} \mathrm{MgCl}$ or with organozinc reagent $\mathrm{PhCH}_{2} \mathrm{ZnCl}$ in THF and diethyl ether, the starting material was recovered unreacted.

Well-documented literature precedence ${ }^{16}$ exists for the nucleophilic ring opening of epoxides. Epoxides 8-10 were reluctant to ring open under the acidic conditions used previously. ${ }^{4,17}$ Epoxide 8 was hydrolyzed to give 1-hydroxy-1,3-diphenylpropan-2-one 12 (50\%) on heating neat in $3 \mathrm{~N}$ sulfuric acid solution at $70{ }^{\circ} \mathrm{C}$ for $3 \mathrm{~h}$ (Scheme 2). Epoxide 9 survived under these conditions, but decomposed when refluxed in THF with $30 \% \mathrm{HclO}_{4}$ for $24 \mathrm{~h}^{4}$ 
Epoxide 10 was unaffected by refluxing in $3 \mathrm{~N}$ sulfuric acid solution over $5 \mathrm{~h}$ or in THF with $30 \% \mathrm{HClO}_{4}$ for $36 \mathrm{~h}$.

\section{Conclusions}

Dimethyldioxirane converts 1-and 2-substituted 1-(benzotriazol-1-yl)alkenes into the corresponding epoxides in almost quantitative yields. The rate of epoxidation decreases for alkenes with electron-withdrawing substituents. A benzotriazole group effectively stabilizes oxiranyl anion at temperatures below $-116{ }^{\circ} \mathrm{C}$, thus allowing various functionalization of the epoxides.

\section{Experimental Section}

General Procedures. Melting points were determined on a MEL-TEMP ${ }^{\circledR}$ capillary melting point apparatus equipped with a Fluke 51 digital thermometer, and are uncorrected. ${ }^{1} \mathrm{H}$ and ${ }^{13} \mathrm{C}$ NMR spectra were recorded at $300 \mathrm{MHz}$ and $75 \mathrm{MHz}$ respectively in $\mathrm{CDCl}_{3}$ and referenced to $\mathrm{Me}_{4} \mathrm{Si}$ for the ${ }^{1} \mathrm{H}$ spectra and $\mathrm{CDCl}_{3}$ for the ${ }^{13} \mathrm{C}$ spectra. Tetrahydrofuran was distilled under nitrogen from sodium-benzophenone immediately before use. All reactions with moisture-sensitive compounds were carried out in dry argon atmosphere. Substituted 1-(1-ethenyl)benzotriazoles were prepared according to previously reported procedures. ${ }^{18}$ Dimethyldioxirane solutions were prepared as described previously., 8

\section{General procedure for the oxidation of vinylbenzotriazoles 1a-d}

Vinylbenzotriazole 1 was placed in a dry flask, dissolved in $1.5 \mathrm{M}$ equivalent of DMD solution in dichloromethane and kept at $-20{ }^{\circ} \mathrm{C}$. The reaction was monitored by TLC. The removal of the solvent afforded the product in quantitative yield.

\section{2-(Benzotriazol-1-yl)oxirane (2a).}

Beige plates (from $\mathrm{CH}_{2} \mathrm{Cl}_{2}$ ) $(100 \%) ; \mathrm{mp} 48.5-50.0{ }^{\circ} \mathrm{C} ;{ }^{1} \mathrm{H}$ NMR $\delta 3.42$ (dd, $J=4.4,3.1 \mathrm{~Hz}, 1 \mathrm{H}$ ), $4.40(\mathrm{dd}, J=4.4,1.6 \mathrm{~Hz}, 1 \mathrm{H}), 5.69(\mathrm{dd}, J=3.1,1.6 \mathrm{~Hz}, 1 \mathrm{H}), 7.43(\mathrm{t}, J=7.4 \mathrm{~Hz}, 1 \mathrm{H}), 7.56(\mathrm{t}, J=$ 
$7.1 \mathrm{~Hz}, 1 \mathrm{H}), 7.95(\mathrm{~d}, J=8.4 \mathrm{~Hz}, 1 \mathrm{H}), 8.10(\mathrm{~d}, J=8.4 \mathrm{~Hz}, 1 \mathrm{H}) ;{ }^{13} \mathrm{C}$ NMR $\delta 46.0,60.3,109.6$, 120.4, 124.7, 128.4, 132.5, 145.7. Anal. Calcd for $\mathrm{C}_{8} \mathrm{H}_{7} \mathrm{~N}_{3} \mathrm{O}$ : C, 59.62; H, 4.38; N, 26.07. Found: C, 59.58; H, 4.38; N, 25.86.

\section{2-(Benzotriazol-1-yl)-2-methyloxirane (2b).}

Yellow oil (97\%); ${ }^{1} \mathrm{H}$ NMR $\delta 2.14(\mathrm{~s}, 3 \mathrm{H}), 3.27$ (d, $\left.J=4.5 \mathrm{~Hz}, 1 \mathrm{H}\right), 3.63(\mathrm{~d}, J=4.5 \mathrm{~Hz}, 1 \mathrm{H})$, 7.34-7.44 (m, 1H), 7.46-7.54 (m, 1H), $7.76(\mathrm{~d}, J=8.2 \mathrm{~Hz}, 1 \mathrm{H}), 8.05(\mathrm{~d}, J=8.2 \mathrm{~Hz}, 1 \mathrm{H}) ;{ }^{13} \mathrm{C}$ NMR $\delta 20.8,53.1,68.3,110.8,120.0,124.3,127.9,131.8,145.9$. Anal. Calcd for $\mathrm{C}_{9} \mathrm{H}_{9} \mathrm{~N}_{3} \mathrm{O}$ : C, 61.70; H, 5.18. Found: C, 61.62; H, 5.43

\section{trans-2-(Benzotriazol-1-yl)-3-methyloxirane (2c).}

White prisms (from ether/pentane) $(99 \%)$; mp $66-67{ }^{\circ} \mathrm{C} .{ }^{1} \mathrm{H}$ NMR $\delta 1.60(\mathrm{~d}, J=5.3 \mathrm{~Hz}, 3 \mathrm{H})$, 4.24 (q, $J=5.3 \mathrm{~Hz}, 1 \mathrm{H}), 5.44$ (s, 1H), 7.36-7.44 (m, 1H), 7.46-7.78 (m, 1H), 7.66 (d, J=8.2 $\mathrm{Hz}, 1 \mathrm{H}), 8.07$ (d, $J=8.4 \mathrm{~Hz}, 1 \mathrm{H}) ;{ }^{13} \mathrm{C} \mathrm{NMR} \delta 16.3,54.1,66.0,109.8,120.2,124.5,128.2,132.7$, 146.2. Anal. Calcd for $\mathrm{C}_{9} \mathrm{H}_{9} \mathrm{~N}_{3} \mathrm{O}: \mathrm{C}, 61.70 ; \mathrm{H}, 5.18 ; \mathrm{N}, 23.99$. Found: $\mathrm{C}, 61.93 ; \mathrm{H}, 5.23 ; \mathrm{N}$, 23.97 .

\section{trans-2-(Benzotriazol-1-yl)-3-phenyloxirane (2d).}

White prisms (from ether/ pentane) $(96 \%) ; \operatorname{mp~} 83-84{ }^{\circ} \mathrm{C} ;{ }^{1} \mathrm{H}$ NMR $\delta 5.10(\mathrm{~d}, J=1.5 \mathrm{~Hz}, 1 \mathrm{H})$, $5.68(\mathrm{~d}, J=1.5 \mathrm{~Hz}, 1 \mathrm{H}), 7.40-7.49(\mathrm{~m}, 6 \mathrm{H}), 7.54-7.59(\mathrm{~m}, 1 \mathrm{H}), 7.75(\mathrm{~d}, J=8.5 \mathrm{~Hz}, 1 \mathrm{H}), 8.12$ $(\mathrm{d}, J=8.3 \mathrm{~Hz}, 1 \mathrm{H}) ;{ }^{13} \mathrm{C}$ NMR $\delta 57.7,67.5,109.8,120.4,124.7,126.0$ (2C), 128.4, 128.9 (2C), 129.4, 132.7, 133.6, 146.3. Anal. Calcd for $\mathrm{C}_{14} \mathrm{H}_{11} \mathrm{~N}_{3} \mathrm{O}$ : C, 70.87; H, 4.67; N, 17.71. Found: C, $70.89 ; \mathrm{H}, 4.65 ; \mathrm{N}, 17.73$.

\section{cis-2-(Benzotriazol-1-yl)-3-phenyloxirane (4).}

White needles (from ether/ pentane) (30\%); mp 103-104 ${ }^{\circ} \mathrm{C} ;{ }^{1} \mathrm{H}$ NMR $\delta 4.57(\mathrm{~d}, J=2.7 \mathrm{~Hz}, 1 \mathrm{H})$, $5.82(\mathrm{~d}, J=2.7 \mathrm{~Hz}, 1 \mathrm{H}), 7.02-7.18(\mathrm{~m}, 5 \mathrm{H}), 7.29-7.37(\mathrm{~m}, 1 \mathrm{H}), 7.40-7.48(\mathrm{~m}, 1 \mathrm{H}), 7.67(\mathrm{~d}, J=$ $8.3 \mathrm{~Hz}, 1 \mathrm{H}), 7.97$ (d, $J=8.3 \mathrm{~Hz}, 1 \mathrm{H}) ;{ }^{13} \mathrm{C}$ NMR $\delta 58.6,65.9,110.5,119.9,124.2,126.4$ (2C), 127.9, 128.2 (2C), 128.8 131.2, 133.4, 145.2. Anal. Calcd for $\mathrm{C}_{14} \mathrm{H}_{11} \mathrm{~N}_{3} \mathrm{O}: \mathrm{C}$ 70.87; $\mathrm{H}, 4.67$; $\mathrm{N}$, 17.71. Found: C, 70.89; H, 4.65; N, 17.73. Found: C, 70.57; H, 4.58; N, 17.59.

\section{trans-2,2-Dimethyl-4-(benzotriazol-1-yl)-5-phenyl-1,3-dioxolane (5).}

White prisms (from ether/pentane) (1.4\%); mp 147-149 ${ }^{\circ} \mathrm{C} ;{ }^{1} \mathrm{H}$ NMR $\delta 1.83$ (s, 3H), 1.88 (s, $3 \mathrm{H}), 6.40$ (d, $J=6.1 \mathrm{~Hz}, 1 \mathrm{H}), 6.61$ (d, $J=6.1 \mathrm{~Hz}, 1 \mathrm{H}), 7.40-7.62(\mathrm{~m}, 6 \mathrm{H}), 7.63-7.72(\mathrm{~m}, 1 \mathrm{H})$, $7.78(\mathrm{~d}, J=8.2 \mathrm{~Hz}, \mathrm{H}), 8.26(\mathrm{~d}, J=8.2 \mathrm{~Hz}, \mathrm{H}) ;{ }^{13} \mathrm{C}$ NMR $\delta 26.4,27.8,80.3,90.6,110.3,112.9$, 120.5, 124.8, 126.4, 128.3, 129.1, 129.2, 133.7, 137.1, 146.8. Anal. Calcd for $\mathrm{C}_{17} \mathrm{H}_{17} \mathrm{~N}_{3} \mathrm{O}_{2}$ : C, 69.14; H, 5.80; N, 14.23. Found: C, 69.17; H, 5.37; N, 13.90. 


\section{Procedure for oxidation of vinylbenzotriazole (1e) 1e}

$(1.5 \mathrm{~g}, 5.04 \mathrm{mmol})$ was dissolved in dimethyldioxirane solution in dichloromethane $(0.22 \mathrm{M}, 28$ $\mathrm{mL}, 6.05 \mathrm{mmol}$ ) and kept at $0{ }^{\circ} \mathrm{C}$. After $18 \mathrm{~h}$, the solvent was removed and another portion of DMD (4.0 mmol, $16 \mathrm{~mL}, 0.27 \mathrm{M}$ ) was added. After another 12 hours, the solvent was removed to give a yellow oil. The product was purified by column chromatography using diethyl ether and pentane to give $\mathbf{2 e}$ and $\mathbf{3 .}$

\section{2-(Benzotriazol-1-yl)-2,3-diphenyloxirane (2e).}

White prisms (from ether/pentane, 52\%); mp 132-133 ${ }^{\circ} \mathrm{C} ;{ }^{1} \mathrm{H}$ NMR $\delta 4.64(\mathrm{~s}, \mathrm{H}), 6.96-7.02(\mathrm{~m}$, 2H), 7.08-7.25 (m, 3H), 7.24-7.28 (m, 3H), 7.30-7.48 (m, 4H), $7.63(\mathrm{~d}, J=8.3 \mathrm{~Hz}, 1 \mathrm{H}), 8.01$ $(\mathrm{d}, J=8.2 \mathrm{~Hz}, 1 \mathrm{H}) ;{ }^{13} \mathrm{C}$ NMR $\delta 68.5,74.4,110.6,120.0,124.2,125.4$ (2C), 126.1 (2C), 128.1, 128.2 (2C), 128.9 (2C), 128.9, 129.6, 132.4, 133.6, 135.2, 145.2. Anal. Calcd for $\mathrm{C}_{20} \mathrm{H}_{15} \mathrm{~N}_{3} \mathrm{O}$ : C, 76.66; H, 4.82; N, 13.41. Found: C, 76.40; H, 4.67; N, 13.54.

\section{2-(Benzotriazol-1-yl-3-oxide)-2,3-diphenyloxirane (3).}

White prisms (from ether/pentane) (34\%); mp $145-147{ }^{\circ} \mathrm{C} ;{ }^{1} \mathrm{H}$ NMR $\delta 4.56(\mathrm{~s}, 1 \mathrm{H}), 7.10-7.26$ (m, 5H), 7.32-7.44 (6H), 7.50-7.58 (m, 1H), $7.63(\mathrm{~d}, J=8.4 \mathrm{~Hz}, 1 \mathrm{H}), 7.91(\mathrm{~d}, J=8.7 \mathrm{~Hz}, 1 \mathrm{H})$; ${ }^{13} \mathrm{C}$ NMR $867.9,74.6,118.8,115.6,124.6,125.3$ (2C), 126.1(2C), 128.4 (2C), 129.0 (2C), 129.3, 130.0, 130.2, 130.9, 131.6, 134.3, 134.6. Anal. Calcd for $\mathrm{C}_{20} \mathrm{H}_{15} \mathrm{~N}_{3} \mathrm{O}_{2}$ : C, 72.94; $\mathrm{H}, 4.59 ; \mathrm{N}$, 12.76. Found: C, 72.82; H, 4.62; N, 12.66.

\section{General procedure for the lithiation of 2-(benzotriazol-1-yl)oxiranes (2a and 2d)}

Oxirane 2 (1.5 mmol) was placed in an oven dried flask under argon, dissolved in dry THF (10 $\mathrm{mL})$, dry diethyl ether $(10 \mathrm{~mL})$ was added as a co-solvent, and the reaction mixture was cooled to $-116{ }^{\circ} \mathrm{C}$ with stirring. To this, 1.1 equiv of LDA (fresh) was added dropwise, and immediately after, an electrophile $(1.65 \mathrm{mmol})$ was added dropwise. The reaction mixture was allowed to warm up to room temperature and quenched with water. The reaction mixture was extracted with $\mathrm{CH}_{2} \mathrm{Cl}_{2}$, dried over $\mathrm{MgSO}_{4}$, and concentrated to give a brown oil, which was purified by column chromatography on silica gel using diethyl ether and pentane.

\section{2-(Benzotriazol-1-yl)-2-trimethylsilyloxirane (6).}

Yellow oil (30\%); ${ }^{1} \mathrm{H}$ NMR $\delta 0.21(\mathrm{~s}, 9 \mathrm{H}), 3.19$ (d, $\left.J=5.0 \mathrm{~Hz}, 1 \mathrm{H}\right), 3.45$ (d, $\left.J=5.0 \mathrm{~Hz}, 1 \mathrm{H}\right)$, $7.25-7.42(\mathrm{~m}, 1 \mathrm{H}), 7.30-7.52(\mathrm{~m}, 1 \mathrm{H}), 7.68(\mathrm{~d}, J=8.4 \mathrm{~Hz}, 1 \mathrm{H}), 8.04(\mathrm{~d}, J=8.2 \mathrm{~Hz}, 1 \mathrm{H}) ;{ }^{13} \mathrm{C}$ NMR $\delta-3.4,50.0,64.2,111.0,120.0,124.2,127.6,132.6,145.6$. 


\section{2-(Benzotriazol-1-yl)-2-benzyl-3-phenyloxirane (8).}

Pale yellow oil (51\%); ${ }^{1} \mathrm{H}$ NMR $\delta 3.32(\mathrm{~d}, J=14.6 \mathrm{~Hz}, 1 \mathrm{H}), 3.53(\mathrm{~d}, J=14.6 \mathrm{~Hz}, 1 \mathrm{H}), 4.75$ (s, $1 \mathrm{H}), 6.75(\mathrm{~d}, J=6.5 \mathrm{~Hz}, 2 \mathrm{H}), 6.94-7.10(\mathrm{~m}, 3 \mathrm{H}), 7.22-7.56(\mathrm{~m}, 6 \mathrm{H}), 7.61(\mathrm{~d}, J=7.0 \mathrm{~Hz}, 2 \mathrm{H})$, 7.95-8.05 (m, 1H); ${ }^{13} \mathrm{C}$ NMR $836.0,64.0,76.0,110.8,119.8,124.1,126.7$ (2C), 127.2, 127.8, 128.3 (2C), 128.8 (2C), 129.0, 129.4 (2C), 132.5, 132.8, 133.7, 145.5. HRMS(FAB) Calcd for $\mathrm{C}_{21} \mathrm{H}_{17} \mathrm{~N}_{3} \mathrm{O}(\mathrm{M}+1): 328.1450$. Found: 328.1457.

2-(Benzotriazol-1-yl)-2-p-toluoyl-3-phenyloxirane (9). White amorphous solid (56\%); mp $118-120^{\circ} \mathrm{C} ;{ }^{1} \mathrm{H}$ NMR $\delta 2.21(\mathrm{~s}, 3 \mathrm{H}), 5.76(\mathrm{~s}, 1 \mathrm{H}), 7.01(\mathrm{~d}, J=8.0 \mathrm{~Hz}, 2 \mathrm{H}), 7.21-7.41(\mathrm{~m}, 4 \mathrm{H})$, $7.48-7.56(\mathrm{~m}, 3 \mathrm{H}), 7.71(\mathrm{~d}, J=8.2 \mathrm{~Hz}, 1 \mathrm{H}), 7.81(\mathrm{~d}, J=7.5 \mathrm{~Hz}, 2 \mathrm{H}), 8.02(\mathrm{~d}, J=8.1 \mathrm{~Hz}, 1 \mathrm{H})$; ${ }^{13} \mathrm{C}$ NMR $821.7,62.8,74.0,110.3,120.2,124.9,126.5$ (2C), 128.6 (2C), 129.0, 129.3 (2C), 129.4 (2C), 130.5, 130.9, 133.1, 145.8, 146.1, 186.3. Anal. Calcd for $\mathrm{C}_{22} \mathrm{H}_{17} \mathrm{~N}_{3} \mathrm{O}_{2}$ : C, 74.35; $\mathrm{H}$, 4.82; N, 11.82 .35; Found: C, 74.04; H, 5.03; N, 11.57 .

\section{2-(Benzotriazol-1-yl)-2-(1,1-diphenyl-1-hydroxymethyl)-3-phenyloxirane (10).}

White amorphous solid (52\%); mp $143-145^{\circ} \mathrm{C} ;{ }^{1} \mathrm{H}$ NMR $\delta 4.50$ (s, H), 4.72 (s, H), 6.90-7.10 (m, 10H), 7.25-7.35 (m, 4H), 7.38-7.48 (m, 1H), 7.49-7.54 (m, 2H), 7.84-7.95 (m, 2H); ${ }^{13} \mathrm{C}$ NMR 864.6, 79.2, 80.8, 112.0, 119.8, 124.3, 126.0 (2C), 126.5 (2C), 127.1, 127.2 (2C), 127.3, 127.4, 127.6 (2C), 127.8 (2C), 128.0 (2C), 128.1, 130.9, 133.2, 140.0, 143.9, 144.7. Anal. Calcd for $\mathrm{C}_{27} \mathrm{H}_{21} \mathrm{~N}_{3} \mathrm{O}_{2}$ : C, 77.31; H, 5.05. Found: C, 77.24; H, 5.28.

\section{$\alpha$-(Benzotriazol-1-yl)acetophenone (11).}

This compound was obtained from 2d following the procedure for the lithiation of 2benzotriazolyloxiranes, except no electrophile was added and the reaction mixture was quenched with water at rt. White plates (from ethyl acetate/hexanes) $(80 \%) ; \mathrm{mp} 115-117{ }^{\circ} \mathrm{C}$ (lit. ${ }^{19} \mathrm{mp}$ 115-116 $\left.{ }^{\circ} \mathrm{C}\right):{ }^{1} \mathrm{H}$ NMR $\delta 6.10(\mathrm{~s}, 2 \mathrm{H}), 7.30-7.80(\mathrm{~m}, 6 \mathrm{H}), 8.01-8.14(\mathrm{~m}, 3 \mathrm{H}) ;{ }^{13} \mathrm{C}$ NMR $\delta 59.3$, $109.5,120.1,124.1,127.8,128.3,129.1,133.8,134.0,134.5,146.0,190.3$.

\section{Procedure for the hydrolysis of oxirane 8.}

Compound $8(0.18 \mathrm{~g}, 0.55 \mathrm{mmol})$ was heated at $70^{\circ} \mathrm{C}$ for $3 \mathrm{~h}$ with $3 \mathrm{~N}$ aqueous solution of $\mathrm{H}_{2} \mathrm{SO}_{4}(30 \mathrm{~mL})$. The reaction mixture was extracted with $\mathrm{CH}_{2} \mathrm{Cl}_{2}$, washed with a sat. solution of $\mathrm{NaHCO}_{3}$, dried over $\mathrm{MgSO}_{4}$, and concentrated to give brown oil. The product was purified by column chromatography on silica gel to give 1-hydroxy-1,3-diphenylpropan-2-one 12 in $50 \%$ yield. 


\section{1-Hydroxy-1,3-diphenylpropan-2-one (12).}

White prisms (from ethyl acetate/hexanes) (50\%); mp 114-115 ${ }^{\circ} \mathrm{C}$ (lit. ${ }^{20} \mathrm{mp} 77-79{ }^{\circ} \mathrm{C}$; lit ${ }^{21} \mathrm{mp}$ $\left.114{ }^{\circ} \mathrm{C}\right) ;{ }^{1} \mathrm{H}$ NMR $\delta 3.64$ (s, 2H), 4.25 (d, $\left.J=4.5 \mathrm{~Hz}, \mathrm{H}\right), 5.19$ (d, $\left.J=4.5 \mathrm{~Hz}, \mathrm{H}\right), 6.95-7.05$ (m, 2H), 7.20-7.50 (m, 8H); ${ }^{13} \mathrm{C}$ NMR $\delta 44.6,79.2,127.2,127.7,128.7,128.9,129.1,129.3,132.8$, 137.5, 206.9.

\section{References}

1. Katritzky, A. R.; Maimait, R; Denisenko, S. N.; Steel, P. J.; Ahkmedov, N. Submitted to J. Org. Chem.

2. Rao, A. S.; Paknikar, S. K.; Kirtane, J. G. Tetrahedron 1983, 39, 2323.

3. Smith, J. G. Synthesis 1984, 629.

4. Katritzky, A. R; Heck, K. A.; Li, J.; Wells, A.; Garot, C. Synth. Commun. 1996, 26(14), 2657.

5. Katritzky, A. R.; Lan, X.; Yang, J. Z.; Denisko, O. V. Chem. Rev. 1998, 98, 409.

6. (a) Katritzky, A. R.; Wu, J.; Kuzmierkiewicz, W.; Rachwal, S. Liebigs Ann. Chem. 1994, 1. (b) Aurrecoechea, J. M.; Lopez, B.; Fernandez, A.; Arrieta, A.; Cossio, F. P. J. Org. Chem. 1997, 62, 1125. (c) Katritzky, A. R.; Qi, M. J. Org. Chem. 1997, 62, 4116. (d) Katritzky, A. R.; Luo, Z.; Fang, Y.; Feng, D.; Ghiviriga, I. J. Chem. Soc., Perkin Tans. 2 2000, 1375.

7. Adam, W.; Bialas, J.; Hadjiarapoglou, L. Chem. Ber. 1991, 124, 2377.

8. Gibert, M.; Ferrer, M.; Sanchez-Baeza, F.; Messeguer, A. Tetrahedron 1997, 53, 8643.

9. Rademacher, P.; Kowski, K.; Katritzky, A. R.; Denisenko, S. N. J. Mol. Struct. 1999, 513, 47.

10. Solladie-Cavallo, A.; Adib, A. Tetrahedron 1992, 48, 2453.

11. Curci, R.; D'Accolti, L.; Dinoi, A.; Fusco, C.; Rosa, A. Tetrahedron Lett. 1996, 37, 115.

12. Eisch, J. J.; Galle, J. E. J. Org. Chem. 1990, 55, 4835.

13. Ashwell, M.; Clegg, W.; Jackson, R. F. W. J. Chem. Soc., Perkin Trans. 1 1991, 897.

14. Florio, S.; Ingrosso, G.; Troisi, L.; Lucchini, V. Tetrahedron Lett. 1993, 34, 1363.

15. Alexakis, A.; Jachiet, D.; Nomant, J. F. Tetrahedron 1986, 42, 5607.

16. Newman, H.; Angier, R. B. Tetrahedron 1970, 26, 825.

17. Katritzky, A. R.; Offerman, R. J.; Cabildo, P.; Soleiman, M. Recl. Trav. Chim. Pays-Bas. 1988, 107, 641 .

18. Katritzky, A. R.; Wu, J. Synthesis 1994, 597.

19. Katritzky, A. R.; Yang, Z.; Lam, J. N. J. Org. Chem. 1991, 56, 6917. 
20. Sohma, A.; Mitsui, S. Bull. Chem. Soc. Jpn. 1970, 43, 448. 